\title{
COMMISSION 20: POSITIONS AND MOTIONS OF MINOR PLANETS, COMETS AND SATELLITES
}

\author{
(POSITIONS ET MOUVEMENTS DES PETITES PLANETES, DES \\ COMETES ET DES SATELLITES)
}

\author{
PRESIDENT: E. Bowell \\ VICE-PRESIDENT: G.B. Valsecchi \\ ORGANIZING COMMITTEE: K. Aksnes, J.-E. Arlot, E. Bowell, A. Carusi, \\ J.A. Fernández, A. Lemaître, B.G. Marsden, H. Rickman, V.A. Shor, \\ G.B. Valsecchi, D.K. Yeomans \& J.-X. Zhang
}

\section{Introduction (E. Bowell)}

The work of Commission 20 has seen a continuing upsurge during the last triennium, in large part due to the enormous number of astrometric positions of asteroids and comets generated by near-Earth asteroid (NEA) search programs. Those programs, and others concerned with the search for transneptunian objects (TNOs), faint outer-planet satellites, and TNO and asteroid satellites, have greatly increased the pool of unusual solar system objects, and have engendered research in hitherto unexplored areas. Rapid maturation of the World Wide Web has also had a beneficial effect on the Commission's work, from the more frequent dissemination of data from the Minor Planet Center, to the ready availability of datasets on asteroid, comet, and satellite orbital elements and ephemerides.

A direct application of the work of our Commission concerns Division III's Working Group on Near-Earth Objects. Discoveries made during the course of the NEA search programs have led to increased work on estimating the true NEA population from the known subset. It was thought that, as of June 2002, about half of NEAs larger than $1 \mathrm{~km}$ diameter (more precisely, brighter than absolute magnitude $H=18$ ) had been discovered. Two recent seminal papers are by Stuart (Science 294, 1691, 2001), who used observations made during the course of the Lincoln Near Earth Asteroid Research (LINEAR) program to estimate the NEA cumulative size distribution down to $H=22 \mathrm{mag}$; and Bottke et al. (Icarus 156, 399, 2002), who, in addition to making a NEA population estimate, studied the source regions for NEAs and calculated the influx rate needed to replenish each region.

There exists an increasing overlap between Commission 20's work and that of Commission 7 (Celestial Mechanics and Dynamical Astronomy) and Commission 15 (Physical Studies of Comets, Minor Planets, and Meteorites). In the former case, we cite the elegant and surprising results of work on asteroid-Earth impact probability, which has in the span of a few years come to seem a routine endeavor. Another example is the "coming of age" of investigations into the Yarkovsky effect, which can cause $\mathrm{km}$-size asteroids to migrate into chaotic resonance zones in the inner main belt, thence onto Earth-approaching orbits (e.g., Bottke, Rubincam, and Burns, Icarus 145, 301, 2000), and can set NEAs onto collision courses with our planet (Giorgini et al., Science, 296, 132, 2002). In the latter case, images of comets taken for astrometric purposes can hardly be separated from their interpretation in terms of cometary physics. Accordingly, we give particular attention in this report to recent results on asteroid orbit determination and the ramifications of cometary orbit determination. Readers are directed to the reports of Commissions 7 and 15 for descriptions of related research. 


\section{Meetings}

Among meetings relevant to Commission 20's work that were held during the interval covered by this report were:

- Asteroids, Comets, Meteors 1999, 26-30 July 1999, Ithaca, NY, USA

- U.S.-European Celestial Mechanics Workshop, 3-7 July 2000, Poznan, Poland

- Asteroids 2001: from Piazzi to the 3rd Millennium, 11-16 June 2001, Palermo, Italy

- Astrometry and Physics of Minor Planets from observational networks, 9-12 October 2001, Paris, France

\section{Asteroids (E. Bowell)}

There have been important developments in the computation of orbits and in identifying and linking asteroid astrometric observations. Largely, these developments have stemmed from the super-exponential growth of the number of observations being reported.

Several new solutions to the inverse problem of orbit computation have been devised, including linear, semilinear, and nonlinear methods. Using linear methods, Chodas and Yeomans (21st Annual AAS Guidance and Control Conference, 1999) developed techniques to incorporate both optical and radar astrometry. Milani (Icarus 137, 269, 1999) discussed the linear approximation using six-dimensional confidence boundaries. Milani also used a semilinear approximation in what he termed a multiple-solution technique, in which the mapping parameter is the step along the principal eigenvector of the covariance matrix. The method is attractive because of its simplicity, and has been applied to work on the collision probability of asteroids with Earth. Virtanen, Muinonen, and Bowell (Icarus 154, 412, 2001) devised a very general approach to orbit estimation that is particularly applicable to short orbital arcs, where orbit computation is almost always nonlinear. Termed statistical ranging, the method uses Monte Carlo selection of orbits in orbital-element phase space. In follow-up work, Muinonen, Virtanen, and Bowell applied statistical ranging to asteroid collision assessment (Celest. Mech. Dynam. Astron. 81, 93, 2001), where they were able to accelerate orbit selection by up to two orders of magnitude, and TNO orbit computation from sparse observational datasets.

In other advances, McNaught (J. Brit. Astron. Assoc. 109, 294, 1999) developed an orbit computation technique based on the variation of topocentric range and the angle to the line of sight; Marsden (Proc. Nautical Almanac Office Sesquicentennial Symp. 333, 1999) discussed a simple generalization of the Väisälä approach that deals particularly effectively with TNOs and NEOs (near-Earth objects) and is used by the Minor Planet Center to prepare uncertainty maps for new discoveries. Bernstein and Khushalani (Astron. J. 120, 3323,2000 ) devised a linearized orbit-fitting procedure in which accelerations are treated as perturbations to the inertial motions of transneptunian objects. The method produces ephemerides and uncertainty ellipses, even for short-arc orbits. Kristensen (Astron. Nachr. $322,47,2001$ ) studied the distribution of observations that would lead to the smallest future ephemeris uncertainty, and also the best time to make observations that would maximally improve an orbit. Although different, Kristensen's results parallel those of Bowell's so-called hierarchical observing protocol (http://asteroid.lowell.edu/cgi-bin/koehn/hop).

Milani (op. cit.) and Chesley and Milani (Paper 99-148 in AAS/AIAA Space Flight Mechanics Meeting, 1999) considered a semilinear method of ephemeris prediction. Making useful predictions for the recovery of essentially lost asteroids was the subject of a paper by Boattini et al. (Astron. Astrophys. 375, 293, 2001). Their method has been applied to make a number of precoveries. Doppler and Gnädig, starting in 1997, developed an automated search technique, and have used it to make about 10000 identifications to date.

Milani and his collaborators have continued their series of papers on establishing identifications among asteroid observations. New identification algorithms have been developed 
by Sansaturio et al. (in Proc. IAU Coll. 172, 363, 1999), Milani et al. (Icarus 144, 39, 2000), and Milani, Sansaturio, and Chesley (Icarus 151, 150, 2001). The algorithms fall into two classes: the first, based on a comparison of orbital elements, has led to a number of so-called orbit identification methods; in the second type, an orbit computed for one of the arcs is used to assess the observations that make up the other arc, and constitutes so-called observation attribution methods.

The determination of asteroid masses through mutual perturbations of pairs of asteroids continues to advance, fueled by the accelerating accumulation of astrometric data. Although the number of asteroid mass determinations has increased, their accuracy has improved only slightly (e.g., Hilton, Astron. J. 117, 1077, 1999; Michalak, Astron. Astrophys. 360, 363, 2000; Michalak, Astron. Astrophys. 374, 703, 2001).

Two additional research areas that are worthy of note depend on an underpinning of asteroid astrometry and accurate orbit computation. The estimation of asteroid profiles through the timing of the occultation of stars, continues to flourish (e.g., Dunham, Sky and Telescope 101, 116, 2001). The computation of a greatly enlarged database of reliable asteroid proper elements by Knežević and Milani (BAAS 31, 1106, 1999) has led to a startling view of the distribution of taxonomic classes in the main asteroid belt from Sloan Digital Sky Survey colorimetry (Ivezić et al.); and to the discovery, within the Koronis family, of the tightly knit Karin cluster, whose age has been constrained to $5.8 \pm 0.2 \mathrm{My}$ (Nesvorný et al., Nature 417, 720, 2002).

\section{Minor Planet Center and Institute for Applied Astronomy (B. G. Marsden)}

\subsection{Minor Planet Center}

The surge in activity so evident during the 1996-1999 triennium escalated to even higher levels during 1999-2002. The columns in the following tabulation show, for each six-month interval, the number of observations, of new provisional designations given to initially unidentified objects (in this case representing the situation as of the end of Sept. 2002), of objects for which new orbits were computed, of new permanent numberings and of new namings of minor planets:

$\begin{array}{lrrrrr}\text { Dates } & \text { Obs. } & \text { Desigs. } & \text { Orbits } & \text { Numbers } & \text { Names } \\ \text { 1999 July-Dec. } & 592287 & 32778 & 5936 & 1985 & 356 \\ \text { 2000 Jan.-June } & 1660416 & 34095 & 25527 & 2697 & 483 \\ \text { 2000 July-Dec. } & 1515800 & 37655 & 24257 & 4242 & 388 \\ \text { 2001 Jan.-June } & 1755398 & 18370 & 16682 & 6163 & 584 \\ \text { 2001 July-Dec. } & 2367865 & 53649 & 33563 & 6656 & 442 \\ \text { 2002 Jan.-June } & 3781865 & 29612 & 29630 & 10992 & 594\end{array}$

It is worth noting that the number of observations published during the most recent half-year was 57 percent greater than the total number of observations in the Minor Planet Center's files as of mid-1999, and that the new numberings during this half-year amounted to very slightly more than the total as of mid-1999. The totals as of mid-2002 were: observations, 14076 366; provisional designations, 376689 (of course, almost half of these refer to numbered objects or involve duplications); orbits, 187941 ; permanent numberings, 43721 ; and names, 9576.

While the MPC has still been able to keep up with the increasing work, thanks in no small measure to the acquisition of new computers purchased from gifts made by the Tamkin Foundation to the Smithsonian Astrophysical Observatory, the shortage of funding for staff members is taking its toll. The MPC staff was increased to three (or more precisely 2.5) when T. B. Spahr joined B. G. Marsden and G. V. Williams there in May 2000, but this is still inadequate realistically for the $16 / 7$ operation at which the MPC aims, let alone the $24 / 7$ manned operation some would like. 
The last of the lost numbered minor planets, (719) Albert, was accidentally rediscovered by the Spacewatch program in May 2000, and it is now believed that the positions of all of the numbered minor planets are routinely predictable to better than a very few arcsecexcept presumably on those rather infrequent occasions when one passes extremely close to the Earth.

The following tabulation refers to the number of pages of MPC publications, again in terms of six-month ranges during the triennium. Here the column MPC shows the number of basic Minor Planet Circulars, these being available in both printed and electronic form. The columns MPS and MPO refer to the Minor Planet Circulars (Observation) Supplement and Minor Planet Circulars Orbit Supplement, respectively, these being available only electronically. The final column refers to the Minor Planet Electronic Circulars.

$\begin{array}{lrrrr}\text { Dates } & \text { MPC } & \text { MPS } & \text { MPO } & \text { MPEC } \\ \text { 1999 July-Dec. } & 2218 & 2810 & 0 & 507 \\ \text { 2000 Jan.-June } & 3502 & 8046 & 1670 & 608 \\ \text { 2000 July-Dec. } & 930 & 7096 & 6000 & 598 \\ \text { 2001 Jan.-June } & 1150 & 8100 & 6210 & 561 \\ \text { 2001 July-Dec. } & 1296 & 11180 & 7290 & 748 \\ \text { 2002 Jan.-June } & 1830 & 15926 & 10876 & 820\end{array}$

It should be noted in particular that the MPS, which had accumulated to just 5480 pages between its inception in Oct. 1997 and June 1999, had amassed an enormous 58638 pages by June 2002. With the inception of the MPS, the MPC restricted its inclusion of observations basically to a summary of the number of observations of each object made at each observatory. However, by early 2000 the detailed publication of orbital information was again increasing the size of the monthly MPC batches unacceptably, so the MPO was introduced in May of that year. This allowed the orbital information in the MPC to be restricted to a concise presentation of the discovery information and orbits of the newly numbered minor planets and a list of new identifications. This explains the substantial drop after the second entry in the MPC column and means that the total number of Minor Planet Circulars issued by the end of the triennium was still only 46080 , even though the number of pages of the Orbit Supplement was already then up to 32046 .

All the above publications basically appear monthly, although personnel constraints mean that it is not always possible to accomplish the extensive filing of data necessary to update all the MPC files, and on such occasions it has been traditional to omit the publications. Beginning in Apr. 2001, and in response to requests that observations of mainbelt minor planets be made available more frequently than once a month, arrangements were made to publish the Observation Supplement at two-week, or even one-week intervals. There was no corresponding publication of the asteroid orbits, however, and there was no filing of the asteroid observational or orbital data. This simplified process can obviously also be applied on those occasions when the past practice would have been to omit all publications, and now there are also "minibatches" of Minor Planet Circulars containing all the material that does not relate to observations and orbits of minor planets. It has become apparent, however, that those who have agitated most for the frequent availability of observations have really meant that they want the data to be fully filed, and there is no way this filing can be accomplished without significant augmentation of the MPC staff.

The Minor Planet Electronic Circulars, of which some 5948 have been issued since their inception in 1993, have principally been used to provide information about individual objects of particular interest. These include in particular transneptunian and nearEarth objects, both when they are first discovered and when observations become available from a second opposition (whether as a recovery, precovery or identification). There are also "Daily Orbit Update" issues prepared automatically during the early morning hours at the MPC containing new identifications found and orbits computed the previous day, as well as new observations of NEOs. The initial MPECs for new NEOs usually follow a period of time (typically 24-36 hours) while the objects concerned (as well as candi- 
dates that turned out not to be NEOs) have been on "The NEO Confirmation Page" at http://cfa-www.harvard. edu/iau/NEO/ToConfirm.html. Since early 1999, confirmed NEOs have subsequently been routinely examined at the University of Pisa, and since early 2002 independently at the Jet Propulsion Laboratory, for Earth-impact possibilities during the next 80-100 years. Possible impact cases are listed on the web pages of CLOMON2 (at http://newton.dm.unipi.it/cgi-bin/neodys/neoibo?riskpage:0;main) and SENTRY (http://neo.jpl.nasa.gov/risks), and these are progressively updated using the observations in the daily orbital update MPECs until the point is reached (unless we are terribly unlucky...) that impact is no longer possible. Typically there are at any time some three dozen NEOs with nonzero impact possibilities during the next century. Most of these are small objects that were lost rather soon after discovery.

\subsection{Minor Planet Names}

The Committee on Small Body Nomenclature has been concerned that the recent proliferation of numberings of minor planets would have an uncontrolled effect on the rate of proposals of new namings. So far the namings have continued to average under 100 per month, and any significant increase in the naming rate is expected to meet with some opposition. In late 2001 the CSBN carried out, with mixed success, an experiment in which there was a vote for the "best" names.

\subsection{Distant Objects}

The number of known "distant objects" rose from 135 in mid-1999 to 679 in mid-2002. The number observed at multiple oppositions rose from 61 to 333 during this time, a slight increase in the "success" rate to now almost half. Although (2060) Chiron, the first centaur, received its permanent number already in 1978, the first numbering of a transneptunian object did not occur until 2000 with (15760) $1992 \mathrm{QB}_{1}$. Since then, the number of numbered centaurs has increased to at least 8 , and the 35 numbered transneptunians include five scattered-disk objects (SDOs), while the three objects (29981) $1999 \mathrm{TD}_{10},(33128) 1998$ $\mathrm{BU}_{48}$ and (42355) $2002 \mathrm{CR}_{46}$ are hybrid cases with mean distances greater than and perihelion distances significantly smaller than those of Neptune. The 333 multiple-opposition distant objects divide into 22 centaurs, 260 "basic" transneptunians, 11 hybrids and 40 SDOs-although 11 of the SDOs have perihelia larger than the canonical $38 \mathrm{AU}$ (up to more than $44 \mathrm{AU}$ in the case of $2000 \mathrm{CR}_{105}$ ).

\subsection{Comets}

Detailed information about comet observations during the triennium is contained in the report of Commission 6 . In addition, it can be noted that the number of numbered comets (i.e., those observed at multiple perihelion passages) increased during the triennium from 140 to 152. Prior to the Minor Planet Center's move from Cincinnati to Cambridge in 1978 it was traditional to publish astrometric observations of comets in the IAU Circulars rather routinely. Except for new discoveries, this practice was inefficient and not particularly appropriate, so it seemed to make sense to publish the comet observations systematically in the Minor Planet Circulars each month. In recent years, some have felt they needed the observations more quickly, and arrangements were made, starting in February 2002, to publish them preliminarily in the Minor Planet Electronic Circulars, essentially on a weekly basis. The sticking point has been to do this reasonably efficiently and in such a way that credit is properly given to the observers.

\subsection{Institute of Applied Astronomy}

The greater-than-exponential numbering rate has also had a significant effect on the operation of the group at the Institute of Applied Astronomy in St. Petersburg that is responsible for the publication of the annual volume of Efemeridy Malykh Planet. This group consists 
of O. M. Kochetova, V. A. Shor, N. K. Sumzina, O. O. Vasil'kova and T. A. Vinogradova, with G. A. Netsvetaeva, E. Yu. Parijskaya and D. A. Ryzhkova responsible for the typesetting. The number of EMP pages increased from 948 in 2000 to 1061 in 2001 to 1295 in 2002 , this being somewhat contained by increasing the number of ephemerides per page from 12 to 14 to 18 . There has also been a version of the ephemerides available on diskette, and for 2002 there has been an arrangement to obtain files of "Updated Ephemerides of Minor Planets" by ftp.

\section{Comets (J. A. Fernández and G. B. Valsecchi)}

The dynamical evolution of the transneptunian region was studied by Petit, Morbidelli and Valsecchi (1999, Icarus 141, 367) with the aim of explaining features such as the heating of the belt in the region at 40-50 AU; they attributed this feature to gravitational interactions with massive bodies scattered by the Jovian planets after their formation. De la Fuente Marcos and de la Fuente Marcos (Astron. Astrophys. 371, 1097, 2001) have modeled early TNO populations under the assumption that the Sun was born in a typical open cluster. They found that the strong perturbations of member stars could have been responsible for the scattered TNO population.

The delivery of icy bodies to the primitive Earth to explain the D/H ratio in the Earth's oceans was studied by Morbidelli et al. (Meteorit. Planet. Sci. 35, 1309, 2000), who found that ice-rich bodies from the outer asteroid belt were the main contributors to the Earth's water, with a late veneer supplied by bodies from the Uranus-Neptune region and from the transneptunian region.

Work concerning Oort cloud dynamics has focused on possible signatures of the distribution of aphelion points of long-period comets, attributed either to close stellar passages (Dybczyński, Astron. Astrophys. 383, 1049, 2002), or to a planet-sized or substellar companion (Murray, Mon. Not. R. astron. Soc. 309, 31, 1999; Matese, Whitman, and Whitmire, Icarus 141, 354, 1999; Matese and Lissauer, Icarus 157, 228, 2002). Serafin and Grothues (Astron. Nachr. 323, 37, 2002) studied in detail the effects of individual stellar encounters on the orbits in the cloud. Stern and Weissman (Nature 409, 589, 2001) analyzed how the dynamical time scale for ejection of residual planetesimals in the region of the Jovian planets compares with the time scale for mutual catastrophic collisions. They found that collisions will prevent most comets escaping from most locations in the region of the Jovian planets, so the total mass that reached the Oort cloud may be smaller than currently believed.

The capture of Oort cloud comets into short-period orbits has been the subject of active research, with several groups examining various aspects of this problem. Levison, Dones and Duncan (Astron. J. 121, 2253, 2001) attempted a global approach, finding that the observed population of Halley-type comets can be explained starting from an Oort cloud consisting of an isotropic outer cloud and a disklike massive inner cloud. Moreover, Levison et al. (Science 296, 2212, 2002) found that, to reconcile their model computations predicting a large number of dormant Halley-type comets with the small number of actual discoveries of these objects, it is necessary to invoke a very high rate of physical disruption of comets evolving inward from the Oort cloud. Nurmi (Mon. Not. R. astron. Soc. 323, 911,2001 ) compared orbital computations made using a Monte Carlo method and a direct integration method, and discussed ways to minimize the discrepancies. Nurmi, Valtonen, and Zheng (Mon. Not. R. astron. Soc. 327, 1367, 2001) studied the time variation of planetary impact probability, assuming that the flux of Oort cloud comets is modulated by the motion of the Sun with respect to the Galactic plane. The study by Rickman, Valsecchi, and Froeschlé (Mon. Not. R. astron. Soc. 325, 1303, 2001) focused on the first stage of the dynamical evolution of Oort cloud comets entering the planetary region for the first time. They found that two processes of comparable importance govern the decrease in semimajor axis: planetary close encounters, especially with Jupiter, and indirect perturbations due to the shifting of the motion from barycentric to heliocentric and back. 
Other studies concerning long-period comets are those by Królikowska (Astron. Astrophys. 376, 316, 2001), showing how the inclusion of nongravitational forces in the computation of cometary orbits having hyperbolic original osculating elements almost always allows one to conclude that such comets are in fact coming in elliptic original orbits, and by Dybczyński (Astron. Astrophys. 375, 643, 2001), who studied numerically the dynamical history of a large number of observed long-period comets.

A study of short-period comets by Levison et al. (Icarus 143, 415, 2000) addressed the impact rates of low-inclination comets on planetary surfaces, while Dones et al. (Icarus 142, 509, 1999) compared the results of Monte Carlo computations based on Öpik equations with those obtained through direct numerical integration. Koon et al. (Celest. Mech. Dynam. Astron. 81, 27, 2001) published a detailed mathematical study of the complicated dynamics of very low-velocity encounters that some peculiar short-period comets undergo with Jupiter.

Concerning the modelling of nongravitational forces, Królikowska, Sitarski and Szutowicz (Astron. Astrophys. 368, 676, 2001) studied the motion of six erratic short-period comets, while Szutowicz (Astron. Astrophys. 363, 323, 2000) studied in detail comet 43P/Wolf-Harrington.

Finally, a comprehensive review of the dynamics of near-Earth objects was published by Gladman, Michel, and Froeschlé (Icarus 146, 176, 2000); they found no dynamical reason to demand that any significant component of the NEO population must come from a cometary source, although such a contribution could not be ruled out.

\section{Satellites (J.-E. Arlot)}

\subsection{New Satellites}

Prior to the XXIVth IAU General Assembly the announcement of new planetary satellites was carried solely by the IAU Circulars issued by the Central Bureau for Astronomical Telegrams (CBAT). With the recent availability of large-format CCD arrays, a number of observing groups are now searching for new small planetary outer satellites that were beyond the reach of earlier photographic surveys. What started with the July 1999 discovery of three new Uranian satellites (IAUC 7230, IAUC 7248), following two 1997 Uranian satellite discoveries, has now become a flood, with an additional 23 Jovian and 12 Saturnian outer satellites. The requirement of publishing the astrometry and orbits for all these objects would have overwhelmed the IAU Circulars. At the XXIVth GA, it was decided that the IAU Circulars would henceforth contain just a summary of the discovery (because it is the CBAT that provides the designations). The Minor Planet Electronic Circulars and Minor Planet Circulars (published by the Minor Planet Center) would then carry the early astrometric observations and preliminary orbit computations, with later data also being archived in the latter.

Three new Uranian satellites had already been numbered and named UXVIII (Prospero), UXIX (Setebos), and UXX (Stephano) at the XXIVth General Assembly; they are discussed in detail by Gladman et al. (Nature 412, 163, 2001).

The 23 new Jovian satellites comprise the objects designated S/2000 J 1-12 and S/2001 $\mathrm{J} 1-11$. Of the 2000 set, all but S/2000 J 11 have been observed at more than one opposition, $\mathrm{S} / 2000 \mathrm{~J} 1$ in fact being identical with the lost S/1975 J 1. The 2001 set are still singleopposition objects. The new Saturnian satellites, designated S/2000 S 1-12, were all also observed at their following opposition.

The latest orbits by the Minor Planet Center for the 38 new satellites are contained on MPC 41900, 42318, 43760-43761, 44032-44033, 44184-44185, 44506, 44862, 45184, 45335 and 45658-45659. Preliminary and improved orbits have also been computed at the Jet Propulsion Laboratory by Jacobson (BAAS 33, 1191, 2001). 


\subsection{Astrometric Observations}

U.S. Naval Observatory. CCD observations of JV (Amalthea), JXIV (Thebe), SXII (Helene), SXIII (Telesto), and SXIV (Calypso), and IR observations of Amalthea, Thebe, JXVI (Metis), JXV (Adrastea), UV (Miranda), and NI (Triton), were obtained using the 61-inch Astrometric Reflector at Flagstaff, during 1992-2000, and were reduced and sent to JPL for ephemeris development (Pascu et al.).

Observations of close approaches of pairs of the Galilean satellites of Jupiter were observed with the speckle camera and the 26-inch telescope, in preparation for observing "mutual phenomena" astrometrically in 2003. (Mason et al., BAAS 31, 1225, 1999).

Pulkovo Observatory (Pulkovo, Russia). The results of photographic observations of Saturn satellites using the 26 -inch refractor at Pulkovo in 1994-1998 were published by Kisseleva and Kalinichenko (Izvestiia Glavnoi Astronomicheskoi Observatorii Pulkovo 214, 344, 2000). These results include 292 mutual positions of the satellites SII (Enceladus), SIII (Tethys), SIV (Dione), SV (Rhea), SVI (Titan), and SVIII (Iapetus).

Measurements of the relative positions of Saturn and Jupiter satellites from 1995 to 2000 , using a CCD camera at the 26-inch refractor at Pulkovo Observatory, were published by Kisseleva and Izmailov (Izvestiia Glavnoi Astronomicheskoi Observatorii Pulkovo 214, $333,2000)$. The relative coordinates of 74 observations of pairs of Saturn satellites and eight pairs of Jupiter satellites were tabulated.

As a result of the revision of observations of Saturn satellites made in Abastumani Astrophysical Observatory using the Double Wide-Field astrograph in 1984, four positions of SVII (Hyperion) and 20 positions of SIV (Dione), SV (Rhea), SVI (Titan), and SVIII (Iapetus) were produced relative to the ACT catalogue (Kisseleva and Chanturiya, Izvestiia Glavnoi Astronomicheskoi Observatorii Pulkovo 214, 356, 2000).

Astrometric observations of SVI (Titan, 4 positions), SVII (Hyperion, 10 positions), SVIII (Iapetus, 11 positions), and SIX (Phoebe, 5 positions) were made at Pulkovo Observatory using the ZA-320 Mirror Astrograph in 1998-1999, and the results were published by Devyatkin et al. (Izvestiia Glavnoi Astronomicheskoi Observatorii Pulkovo 214, 382, 2000).

Goloseevo Observatory (Kiev, Ukraine). CCD observations of the close satellites of Jupiter were made using the 2-meter telescope and Two-Channel Focal Reducer at Terskol Observatory (North Caucasus) in 1998-2000. From these observations, 64 positions of JV (Amalthea), 84 positions of JXIV (Thebe), 42 positions of JXV (Adrastea), and 128 positions of JXVI (Metis) were published by Ledovskaya et al. (Kinematika i Fizika Nebesnykh Tel. 15, 483, 1999).

Nikolaev Observatory (Nikolaev, Ukraine). Observations of the Galilean satellites made in Nikolaev in 1962-1998 were processed using the PPM catalog and the HIPPARCOS and ACTRC star catalogues. 1314 absolute positions were published.

Bordeaux Observatory (France). Observations were continued on the bright satellites of Saturn using the transit circle instrument of Bordeaux Observatory equipped with a CCD. Using TASS ephemerides, positions of Saturn were deduced. Positions of SVII (Hyperion) and SVIII (Iapetus) were observed and compared with the theory of Duriez and Vienne.

Observations of Titania and Uranus were made during spring and summer 2001 to prepare for the observation of an occultation of a Hipparcos star by UIII (Titania). An offset in the ephemerides was deduced from these observations.

Institut de Mécanique Céleste/Bureau des Longitudes (Observatoire de Paris, France). During the interval 1999-2002, a campaign of observations of the outer satellites of Jupiter and Saturn was organized at the Observatoire de Haute-Provence. 163 observations of JVI (Himalia), 165 of JVIII (Elara), 185 of JVIII (Pasiphae), 165 of JIX (Sinope), 156 of JX 
(Lysithea), 156 of JXI (Carme), 155 of JXII (Ananke), 166 of JXIII (Leda), 94 of S/1999 $\mathrm{J} 1,45$ of S/2000 S 3 and 413 of SIX (Phoebe) were made (Fienga et al., Astron. Astrophys. $391,767,2002)$. In addition, 160 positions of the Galilean satellites were observed and 99 of the bright satellites of Saturn. NII (Nereid) was also observed.

The catalogue of the 1995 observations of mutual events of the satellites of Saturn was published (Thuillot et al., Astron. Astrophys. 371, 343, 2001).

Observations of UXV (Puck) were made using adaptive optics at the European Southern Observatory (Descamps et al., C.R.A.S. 3, 121, 2001; Descamps et al., BAAS 32, 1078, 2000).

The inner satellites of Jupiter, JV (Amalthea) and JXIV (Thebe), were observed at Pic du Midi Observatory, France.

Rio de Janeiro Observatory (Brazil). Campaigns of observations of the planetary satellites were continued using a coronagraph with a variable diameter occulting disk (Bourget, Veiga, Vieira-Martins, PASP 113, 436, 2001). Additional observations were published: photographic observations of Helene (Veiga and Vieira-Martins, Astron. Astrophys. Suppl. 143, 405. 2000); photographic positions of the first eight satellites of Saturn (Veiga and Vieira-Martins, Astron. Astrophys. Suppl. 139, 305, 1999); CCD observations of Phoebe (Veiga, Vieira-Martins, and Andrei, Astron. Astrophys. Suppl. 142, 81, 2000); CCD observations of the satellites of Uranus (Veiga and Vieira-Martins, Astron. Astrophys. Suppl. 138, 247, 1999); and CCD observations of NII (Nereid) (Veiga, Vieira-Martins, and Le Guyader, Astron. Astrophys. Suppl. 136, 445, 1999).

\subsection{Processing of Observations}

Franklin and Aksnes (Astron. J. 122, 2734, 2001) analyzed some 500 observations of mutual events of the Galilean satellites between 1908 and 1997 and found clear evidence for a secular acceleration in Io's motion. The results imply that Io is now spiraling slowly inward, losing more orbital energy from internal dissipation than it gains from Jupiter's tidal torque.

Sternberg Astronomical Institute (Lomonosov University, Moscow, Russia). An original approach was devised and developed by Emel'yanov et al. (Astron. Astrophys. Suppl. $141,433,2000$ ) to process photometric observations of the natural satellites during their mutual occultations and eclipses. The photometric data are reduced to the planetocentric rectangular coordinate differences of satellite pairs. With this method a treatment of the photometric observations of the Galilean satellites of Jupiter during their mutual occultations and eclipses in 1997 on the observatories of Kazakh Republic, Russia, and Ukraine, was made, resulting in 44 mutual planetocentric positions of satellites.

Institute of Astronomy of Catania University (Catania, Italy). During the interval July 1999 to June 2002 the Catania activity on Natural Planetary Satellites was mainly devoted to the analysis of the phenomena observed at M. G. Fracastoro observing station during the PHEMU 97 campaign. Astrometric positions were derived from 28 lightcurves, and led to a study of the agreement between the predicted and observed characteristics of the lightcurves (Blanco, Riccioli, and Cigna, Planet. Space Sci. 49, 31, 2001).

U.S. Naval Observatory. HST astrometric observations of four inner satellites of Neptune, made in 1997, have been analyzed, and new mean motions for NVIII (Proteus), NVII (Larissa), NVI (Galatea), and NV (Despina) were determined. A publication of the results is being prepared (Pascu et al., BAAS 31, 1229, 1999; Pascu, Rohde et al., BAAS 31, 1093, 1999).

Institut de Mécanique Céleste/Bureau des Longitudes (Observatoire de Paris, France). Mutual events of Saturn satellites were subject to a preliminary analysis (Arlot, Thuillot, 
and Ruatti, Celest. Mech. Dyn. Astron. 81, 193, 2002). An analysis of CCD observations of mutual events of Saturn satellites was made, and direct positions were deduced (Vienne et al. Astron. Astrophys. 380, 727, 2001). Available observations of SIX (Phoebe) were fitted to the theory to provide a new ephemeris. An analysis of observations of the inner satellites of Jupiter was carried out (Vachier et al., CELMEC III wokshop, Rome).

\subsection{Ephemerides}

Sternberg Astronomical Institute (Lomonosov University, Moscow, Russia). The circumstances of mutual occultations and eclipses of Jupiter's Galilean satellites in 2002-2003 were computed (Emel'yanov N.: 2001, Astronomy and Geodesy in the New Millennium. Proceedings of International Conference "AstroKazan-2001", September 24-29, 2001, 111-113).

Predictions of favorable circumstances for observing Saturn's co-orbital satellites SX (Janus) and SXI (Epimetheus), as they pass through superior conjunction from August 20, 2004 to June 1, 2005, were made and published. Circumstances of Jupiter outer-satellite encounters were also computed and published (Emel'yanov, Solar System Res. 35, 209, 2001).

Jet Propulsion Laboratory. The work in the past three years has focused primarily on two areas: support and data analysis for the Galileo mission, and determination of the orbits of the irregular satellites of Jupiter, Saturn, and Uranus.

For the Galileo mission, models of the orbits of the Galilean and inner Jovian satellites have been continually improved. In conjunction with the orbit determination a new gravity field for the Jovian system was developed. The orbits of the Galileans, JV (Amalthea), and Thebe were numerically integrated using an analytical theory for the orbits of JXVI (Metis) and JXV (Adrastea). The integration and theory are fit to an extensive set of Earthbased observations as well as data acquired by the Pioneer, Voyager, Ulysses, Cassini, and Galileo spacecraft. The spacecraft data are crucial to the gravity field development (Anderson et al., BAAS 31, 1181, 1999; Anderson et al., EGS, 2000 Conference, Nice, France; Anderson et al, Icarus 153, 157-161, 2001; Anderson et al., J. Geophys. Res. 106(E12), 32963, 2001; Anderson et al., BAAS 33, 1101, 2001; Jacobson, BAAS 33, 1039, 2001; Burns et al., BAAS 33, 1193, 2001; Schubert et al., BAAS 32, 1046, 2000).

The ephemerides of Jupiter's outer satellites were improved (Jacobson, BAAS 32, 861, 2000; Jacobson, Astron. J. 120, 2679, 2000).

In addition to the above, the satellite software system has been upgraded by maintaining and updating the observations and ephemeris database. Ephemerides for all 101 natural satellites (including the Moon) are provided to JPL and non-JPL users (the latter via the Horizons system).

Institut de Mécanique Céleste/Bureau des Longitudes (Observatoire de Paris, France). Circumstances of the 2002-2003 mutual events of the Galilean Satellites were calculated (Arlot, Astron. Astrophys. 383, 719, 2002), and are available, together with interactive software for calculating local observing conditions, on the IMCCE web site (http://www.imcce.fr /Phemu03/phemu03_eng.html) Mutual events involving JV (Amalthea) are also available, as are ephemerides of newly discovered outer satellites of Jupiter and Saturn.

Institute of Applied Astronomy, (St. Petersburg, Russia). Observations of the Galilean satellites made at Nikolaev Observatory from 1962 to 1997 led to an improved ephemeris of Jupiter (Vasilyev et al., Trudy IPA RAN 5, 176, 2000).

\subsection{Theoretical Work}

Institute of Applied Mathematics (Moscow, Russia). A series of theoretical results was published by Vashkov'yak on the evolution of orbits of the new distant irregular satellites 
of Jupiter, Saturn and Uranus. The evolution was mainly investigated by analytically solving Hill's double-averaged problem for the planet-Sun-satellite system and by numerically integrating the averaged equations. Having analyzed the configuration and dynamics of the orbits of Uranus's five outer satellites, the possibility of their mutual crossings became apparent. Vashkov'yak offered a celestial-mechanical explanation for the absence of equatorial satellites of Uranus in the intermediate region between inner near-equatorial satellites and distant irregular satellites. Based on characteristic features of the orbital evolution of Saturn's new satellites, he proposed their natural classification. For new outer satellites of Jupiter, Saturn, and Uranus the extreme eccentricities and inclinations, as well as the circulation periods of the pericenter arguments and of the longitudes of ascending nodes, were determined (Vashkov'yak, Pisma v astronomicheskii zhurnal 27, 470, 2001; 27, 533, $2001 ; 27,543,2001)$.

NAO RAL (U.K.). Numerical integration of the five major Uranian satellites from the ephemerides was carried out. From a spectral analysis of 50 years of data, the major shortperiod perturbations and one of the secular perturbations were identified. The remaining secular terms will have periods longer than the timespan of the observations. The spectral analysis results are being compared with theory.

Institut de Mécanique Céleste/Bureau des Longitudes (Observatoire de Paris, France). A new theory of the motion of the Galilean satellites of Jupiter was made thanks to frequency analysis, and will be applied to make new ephemerides after fitting available observations (Lainey and Vienne, CELMEC III, Rome, Italy, 2001). A new numerical integration was performed for the motion of the satellites of Neptune (Le Guyader). Observations of SI (Mimas) were used to determinate the eccentricity of the orbit of SIII (Tethys) and to understand the Mimas-Tethys commensurability (Vienne, Ceres 2001 Workshop on Astrometry and Physics of Minor Planets from Observational Networks, Paris, 2001; Vienne, CELMEC III, Rome, Italy, 2001).

\subsection{Asteroid Satellites}

An analysis of the observations of several asteroids suspected of binarity was made to detect the signature of a satellite (Thuillot, Ceres 2001 Workshop on Astrometry and Physics of Minor Planets from Observational Networks, Paris, 2001).

Direct observational searches were made (Merline, BAAS 33, 5201, 2001), using adaptive optics (e.g., Close et al., SPIE 4007, 796, 2000), or by analyzing data from the NEARShoemaker spacecraft orbiting (433) Eros (Merline et al., LPI 30, 2055, 1999). A discovery was made of a satellite around (45) Eugenia (named S/1998 (45) 1 "Petit-Prince") (Merline et al., Nature 401, 565, 1999). Lack of astrometric positions precluded the development of models of asteroid satellite motion, and theoretical work was restricted to the formation of such satellites.

\subsection{Miscellaneous}

A review of the possibility of small telescopes for the observation of Natural Planetary Satellites was made (Arlot, Annual Scientific Meeting of the Astronomische Gesellschaft at the Joint European and National Meeting JENAM 2001 of the European Astronomical Society and the Astronomische Gesellschaft in Munich, JD 03 02).

In response to solicitations for input into the National Research Council's Solar System Exploration Decadal Study, for the period 2003-2013, a panel was formed to produce a "White Paper" on the needs of astrometry in the Solar System. (Pascu et al.).

A new scale field in the Pleiades, containing 10 Hipparcos stars and 10 additional Tycho stars (on a $5 \times 7$-inch plate) was identified and used to redetermine the scale of the USNO 26 -inch refractor. The new value agreed with the older value, but a substantial coma term 
was detected. This scale value is to be used for reduction of satellite observations made with the 26-inch. (Pascu et al., BAAS 33, 3, 2001).

\section{Working Group on Natural Planetary Satellites (J.-E. Arlot)}

The WG was chaired by J.-E. Arlot, and its members were K. Aksnes, J.-E. Arlot, C. Blanco, N. V. Emel'yanov, R. A. Jacobson, J. H. Lieske, T. Nakamura, D. Pascu, M. Rapaport, P. K. Seidelmann, D. B. Taylor, R. Vieira-Martins, and G. V. Williams.

The activities of the working group on natural planetary satellites mainly concerned maintenance of the database of astrometric observations of natural planetary satellites:

- Mutual events of the Galilean satellites: Most of the events observed during the past campaigns are available; the lightcurves are available for 1985 and 1991.

- Astrometric observations: most of the observations made in the countries of the former Soviet Union before 1990 are now available.

- Standard data: An effort was made to provide data directly usable for theoretical purposes, such as fitting theories or validation of ephemerides.

- Bibliographic data: All astrometric data are documented through the database of the papers in which they were published. Theoretical papers are also included in the database.

The website of the working group is at http://ww.imcce.fr/iauwg.html. The database is available at http://www.imcce.fr/nsdc.html.

\section{Working Group on Comets and Distant Objects (B. G. Marsden)}

The WG was chaired by B. G. Marsden, and its members were M. F. A'Hearn, M. E. Bailey, A. Gilmore, B. Gladman, D. W. E. Green, S. Isobe, D. Jewitt, B. G. Marsden, K. Muinonen, S. Nakano, J. Parker, E. Roemer, G. Sitarski, S. A. Stern, G. Tancredi, G. B. Valsecchi, C. Veillet, and D. K. Yeomans.

Commission 20's Working Group on Comets expanded its purview in 2000 to include distant objects. While this may be logical from a physical point of view, there is a difference between how the categories are handled astronomically. Furthermore, although some initial ideas were brought up for consideration by members of the Working Group, next to no interest was forthcoming in pursuing them, particularly for the centaurs and transneptunian objects. In the case of the comets, there has been some consideration of improving the definition of "magnitude", given in particular that most astrometric observations are nowadays made using CCDs. The present system involving the use of "total" and "nuclear" magnitudes $m_{1}$ (or $T$ ) and $m_{2}$ (or $N$ ) may have had its value in the photographic era, but it is scarcely applicable now, notably also for predicting magnitudes in ephemerides. A subcommittee of the Working Group has been working on this problem and hopes to be able to present some appropriate recommendations at the upcoming meetings in Sydney.

E. Bowell

President of the Commission 